OPEN ACCESS

Edited by:

Yoshihide Mitani,

Mie University, Japan

Reviewed by:

Elumalai Appachi,

Baylor College of Medicine,

United States

Tomoaki Murakami,

Sapporo Higashi Tokushukai

Hospital, Japan

*Correspondence:

Andrea Emese Jakab jakab.andrea0817@gmail.com; jakab.andrea@med.u-szeged.hu

Specialty section:

This article was submitted to

Pediatric Cardiology,

a section of the journal

Frontiers in Pediatrics

Received: 01 October 2019 Accepted: 08 June 2020 Published: 16 July 2020

Citation: Jakab AE, Hidvégi EV, Illyés $M$, Cziráki A, Kalmár T, Maróti Z and Bereczki C (2020) Childhood Obesity:

Does it Have Any Effect on Young Arteries? Front. Pediatr. 8:389. doi: 10.3389/fped.2020.00389

\section{Childhood Obesity: Does it Have Any Effect on Young Arteries?}

\author{
Andrea Emese Jakab ${ }^{1 *}$, Erzsébet Valéria Hidvégi ${ }^{2}$, Miklós Illyés $^{2}$, Attila Cziráki ${ }^{2}$, \\ Tibor Kalmár ${ }^{1}$, Zoltán Maróti ${ }^{1}$ and Csaba Bereczki ${ }^{1}$
}

${ }^{1}$ Department of Pediatrics, Albert Szent-Györgyi Health Center, University of Szeged, Szeged, Hungary, ${ }^{2}$ Heart Institute, UP Clinical Centre, University of Pécs, Pécs, Hungary

Prevalence of overweight $(\mathrm{OW})$ and obesity $(\mathrm{O})$ in children and adolescents has been increased in the past three decades. Increased arterial stiffness measuring by aortic pulse wave velocity $\left(\mathrm{PWV}_{\mathrm{ao}}\right)$ might be detected in $\mathrm{OW} / \mathrm{O}$ children and adolescents. The aim of our study was to compare the arterial function parameters (AFPs), such as $\mathrm{PWV}_{\mathrm{ao}}$; aortic augmentation index (Aix $\left.\mathrm{x}_{\mathrm{ao}}\right)$; aortic systolic blood pressure $\left(\mathrm{SBP}_{\mathrm{ao}}\right)$ and brachial systolic blood pressure ( $\mathrm{SBP}_{\text {brach }}$ ) measured simultaneously in $\mathrm{O} / \mathrm{OW}$ patients and healthy subjects. In our study 6,816 subjects (3,668 boys) aged 3-18 years were recruited and categorized by their body mass index (BMI) into normal weight (N), OW and $\mathrm{O}$ groups regarding their age and sex. AFPs were measured by a non-invasive, occlusive-oscillometric device. 19.9\% $(n=1,356)$ of the population were OW/O, 911 (516 boys) were OW and 445 (272 boys) were O. After accounting for the effect of covariates, $\mathrm{PWV}_{\text {ao }}$ did not differ significantly between $\mathrm{N}(5.9 \pm 0.8 \mathrm{~m} / \mathrm{s})$ and $\mathrm{OW}$ patients $(5.9 \pm 0.8 \mathrm{~m} / \mathrm{s})$; and $\mathrm{N}(6.0 \pm 0.7 \mathrm{~m} / \mathrm{s})$ and $\mathrm{O}$ patients $(6.0 \pm 0.8 \mathrm{~m} / \mathrm{s})$. Aix ao was significantly lower in OW (9.3 $\pm 7.4 \%$ vs. $7.6 \pm 7.0 \%, p<0.00001)$ and in $O$ patients $(9.7$ $\pm 8.1 \%$ vs. $6.6 \pm 7.2 \%, p<0.00001)$ compared to controls. No significant difference was found regarding $\mathrm{SBP}_{\mathrm{ao}}$ values between controls and $\mathrm{OW}$ and $\mathrm{O}$ groups $(N=110.7$ $\pm 12.4 \mathrm{mmHg}$ vs. $\mathrm{OW}=110.3 \pm 11.9 \mathrm{mmHg} ; N=115.6 \pm 14.0 \mathrm{mmHg}$ vs. $\mathrm{O}=$ $114.3 \pm 12.8 \mathrm{mmHg}$ ). According to our results we may conclude that the unchanged $\mathrm{PWV}_{\mathrm{ao}}$ in $\mathrm{O} / \mathrm{OW}$ subjects might be due to the compensatory decrease in Aixao, referring to enhanced vasodilatory status in the studied population.

Keywords: children and adolescents, overweight, obesity, arterial stiffness, pulse wave velocity, augmentation index, central blood pressure

\section{INTRODUCTION}

Prevalence of overweight $(\mathrm{OW})$ and obesity $(\mathrm{O})$ in children and adolescents has increased in both genders in the past three decades (1). Obesity is associated with significantly decreased life expectancy (2), most cardiovascular (CV) deaths are attributed to OW and $\mathrm{O}(3)$. Obese children are prone to develop early $\mathrm{CV}$ morbidity and are at increased risk for $\mathrm{CV}$ mortality during their adult life (4).

Non-invasive measuring of arterial function parameters (AFPs) - such as aortic pulse wave velocity $\left(\mathrm{PWV}_{\mathrm{ao}}\right)$, aortic augmentation index $\left(\mathrm{Aix}_{\mathrm{ao}}\right)$ and aortic systolic blood pressure $\left(\mathrm{SBP}_{\mathrm{ao}}\right)-$ is a widely accepted and recommended in CV risk stratification in adults (5), as these parameters proved to be strong, independent predictors for CV morbidity and mortality (6-8). 
Numerous works (cc. 400) (9) were published concerning the relationship between childhood OW/O and arterial stiffness. Evaluating these publications several studies were using local stiffness, measuring regional PWV [e.g., carotid (10-12), radial $(13,14)$, and tibial (15)]. However, the local and some regional PWV measurements provide information about only the stiffness at the actual point (carotid) and/or a segment (radial, brachial tibial) of the measurement. Furthermore, only carotid-femoral (cfPWV) or $\mathrm{PWV}_{\text {ao }}$ proved to be a powerful marker to predict the CV morbidity and mortality in adult humans $(8,16)$. Therefore, papers where not $\mathrm{PWV}_{\text {ao }}$ or cfPWV were measured, we considered irrelevant from our aspect. Furthermore, $\mathrm{PWV}_{\mathrm{ao}}$ rises with age, especially after the beginning of puberty in both sexes $(17,18)$. In this age range, the slope of the increase in the $\mathrm{PWV}_{\text {ao }}$ is noticeable, thus merely 2 months difference in age among adolescents shows a marked, significant increase in $\mathrm{PWV}_{\mathrm{ao}}$ (18). Consequently, if we would like to compare $\mathrm{PWV}_{\mathrm{ao}}$ in $\mathrm{OW} / \mathrm{O}$ subjects with their $\mathrm{N}$ (normal weight) pupils, the identical age should be crucial. Obesity in children and adolescents is characterized by chronic sympathetic overdrive. Sympathetic overactivity occurs in obese patients, as evidence of increased heart rate, blood pressure and cardiac output (19). Moreover, AFPs are strongly influenced by the actual brachial systolic blood pressure ( $\mathrm{SBP}_{\text {brach }}$ ) (20), thus $\mathrm{SBP}_{\text {brach }}$ should be the same in the investigated OW/O patients and controls (normal weight, $N$ ). After a careful review of the available scientific literature, where the sex ratio of the studied population was given, the mean age and the $\mathrm{SBP}_{\text {brach }}$ of OW/O patients and control groups was identical, only the most relevant $\mathrm{PWV}_{\mathrm{ao}}$ or cfPWV measurement was performed, no paper fulfilled these rigorous criteria.

Therefore, the aim of our study was to compare the $\mathrm{PWV}_{\mathrm{ao}}$, $\mathrm{Aix}_{\mathrm{ao}}, \mathrm{SBP}_{\mathrm{ao}}$, and $\mathrm{SBP}_{\text {brach }}$ measured simultaneously in $\mathrm{OW} / \mathrm{O}$ patients and healthy subjects in a population of the wide age range (3-18 years), where the patients and control groups were accurately matched by age, sex and $\mathrm{SBP}_{\text {brach }}$ to eliminate their effects on AFPs. We intended to apply an easy-to-use, userindependent occlusive-oscillometric device, which enabled us to expand AFP measurements even into a very young (from 3 years) and large population.

\section{MATERIALS AND METHODS}

\section{Subjects}

6,816 subjects (3,668 boys) aged 3-18 years were recruited from elementary, primary, and high schools in Szolnok town (Hungary) between 2012 and 2016. All the subjects were Caucasian without any chronic diseases, and they were not on any regular medications. Informed consent for the measurements was asked for from the parents of the subjects. The protocol was reviewed and approved by the local Institutional Ethics Committee of the University of Pécs, Pécs, Hungary.

\section{Methods}

Anthropometric measurements (body weight and height) of each subject were carried out according to the WHO recommendation (Kern MGB 150K 100 type electronic scale, MSF 200 type steel strip stadiometer, Kern \& SOHN GmbH, Germany) (21). Subjects were categorized by their body mass index (BMI) into $\mathrm{N}$ (BMI $<90$ percentile), OW (90 percentile $\leq$ BMI $<98$ percentile), and $\mathrm{O}$ (BMI $\geq 98$ percentile) groups regarding their age and sex as well (22).

A non-invasive, occlusive-oscillometric device (Arteriograph, TensioMed Ltd, Budapest, Hungary) was used to the AFPs measurements, for which the operating procedure, in practice, did not differ from a standard digital blood pressure measurement. Clinical use of the Arteriograph is user-independent, painless and takes only 2 min with a onecuff method, hence it is well tolerated by the youngest age group as well. Non-invasive and invasive validations and the detailed method of the device have been published previously $(23,24)$. The device showed the best variance and reproducibility according to comparative studies against applanation tonometry and piezoelectric methods (23). Furthermore, Arteriograph formerly was used in a large cohort to determine the $\mathrm{PWV}_{\mathrm{ao}}$ and $\mathrm{Aix}_{\mathrm{ao}}$ values (percentiles) in a normal, healthy population aged between 3 and 18 years $(18,25)$. Since the device is user-independent, interobserver and intraobserver errors of the sternal notch-pubic bone distance measurements are only relevant, which have been published earlier and proved to be well within acceptable limits (18). Measurements were taken after a 10 -min rest in a comfortable, supine position at room temperature in kindergartens, elementary, and high schools.

\section{Statistics}

Data are reported as mean and SD for continuous data. For statistical tests " $p$ " value $<0.05$ was used for significance. Bivariate correlation was used for testing correlations between the measured parameters and to generate the descriptive statistics of the original data. Statistical analysis was performed with the SPSS 23.0.0.0 statistical package (SPSS Inc., Chicago, Illinois, USA) and $\mathrm{R}$ (version 3.4.4) MatchIt (version 3.0.2) package.

\section{Propensity Score Analysis}

Our data indicated that the distribution of age and sex were significantly different in the different weight categories. Furthermore, strong correlations were observed between the anthropometric, hemodynamic, and arterial function parameters (Supplementary Table 1). To account for the effect of covariates on AFPs propensity score matching was carried out using the "Nearest Neighbor" method with the grouping variable weight (BMI category of $\mathrm{N}, \mathrm{OW}$, and $\mathrm{O}$ ) and $\mathrm{SBP}_{\text {brach }}$, age and sex as variables to match on. The resulting groups contained 911 matched individuals in case of control vs. OW and 445 matched individuals in case of control vs. $\mathrm{O}$ cases that had an almost identical distribution of $\mathrm{SBP}_{\text {brach }}$, age and sex. The plot of the mean of each covariate against the estimated propensity score, separately by weight status is shown in Supplemental Digital Content (Supplementary Figure 1).

\section{RESULTS}

Totally 6,816 subjects (boys $n=3,668 ; 53.8 \%$ ) were involved in this study. Among boys 516 were OW (14.1\%), 272 were O 
(7.4\%), whereas in girls 395 (12.5\%) were OW and $173(5.5 \%)$ were $\mathrm{O}$. The total prevalence of $\mathrm{OW}$ and $\mathrm{O}$ was significantly higher in boys $(21.5 \%)$ than in girls $(18 \%)$ in girls $(p<0.001)$. Altogether $19.9 \%(n=1356)$ of the population were OW/O and $80.1 \%(n=5460)$ of the participants were $\mathrm{N}$.

Baseline characteristics of the investigated cohorts grouped by age and weight categories are summarized for boys (Table 1) and girls (Table 2). Bodyweight, height, $\mathrm{BMI}, \mathrm{SBP}_{\text {brach, diastolic }}$ blood pressure $\left(\mathrm{DBP}_{\text {brach }}\right)$, mean arterial pressure (MAP), pulse pressure (PP) elevated with increasing age in both genders in every weight category, while heart rate (HR) decreased.

Mean $\mathrm{HR}$ and $\mathrm{SBP}_{\text {brach }}$ values showed an increment with increasing BMI categories (Table 3). HR showed a clinically insignificant increased mean value in OW group than in $\mathrm{N}$ subjects $(79.6 \pm 14.1 / \mathrm{min}$ vs. $79.5 \pm 14.9 / \mathrm{min}$, non-significant). However, the HR was significantly increased in $\mathrm{O}$ group compared to $\mathrm{N}$ group $(82.5 \pm 13.5 / \mathrm{min}$ vs. $79.5 \pm 14.9 / \mathrm{min}, p$ $<0.0001)$. The mean HR values were also significantly different in OW and $\mathrm{O}$ groups $(79.6 \pm 14.1 / \mathrm{min}$ vs. $82.5 \pm 13.5 / \mathrm{min}, p<$ $0.02)$. Mean $\mathrm{SBP}_{\text {brach }}$ was higher in $\mathrm{OW}$, and higher in $\mathrm{O}$ patients, than in $\mathrm{N}$ subjects $(N=117.7 \pm 13.1 \mathrm{mmHg}$, OW $=125.6 \mathrm{n}$ $\pm 14.0 \mathrm{mmHg}, \mathrm{O}=131.2 \pm 15.2 \mathrm{mmHg}$ ), the differences were significant between every patient groups $(p<0.0001)$.

Characteristics of the propensity score matched groups and clarified AFPs are summarized in Table 4.

Mean age was significantly lower in O group (11.8 \pm 3.6 ys) compared to OW group $(12.7 \pm 3.8$ ys $)(p<0.001)$. Sex ratio was the same in the investigated groups. The mean body height was almost the same both in OW and $\mathrm{O}$ groups compared to controls, the difference was $0.8 \mathrm{~cm}(\mathrm{NS}, p=0.39)$ and $1.6 \mathrm{~cm}$ (NS, $p=0.28$ ), respectively. Obviously, the mean body weight was significantly higher in $\mathrm{OW}$ and $\mathrm{O}$ groups than in controls. Although the $\mathrm{O}$ patients were younger, their mean $\mathrm{SBP}_{\text {brach }}$ was significantly higher $(131.2 \pm 15.2 \mathrm{mmHg})$ than was in $\mathrm{OW}$ patients $(125.6 \pm 14.0 \mathrm{mmHg})(p<0.0001)$.

After the elimination of the alternating effects of age, sex and $\mathrm{SBP}_{\text {brach }}, \mathrm{PWV}_{\text {ao }}$ values were not significantly different between the propensity score matched $\mathrm{N}$ patients $(5.9 \pm 0.8 \mathrm{~m} / \mathrm{s})$ and $\mathrm{OW}$ patients $(5.9 \pm 0.8 \mathrm{~m} / \mathrm{s})$ and between $N$ patients $(6.0 \pm 0.7 \mathrm{~m} / \mathrm{s})$ and $O$ patients $(6.0 \pm 0.8 \mathrm{~m} / \mathrm{s})$. A significant difference was found between $\mathrm{N}$ and $\mathrm{OW}$ patients in Aix $\mathrm{xa}_{\mathrm{ao}}$, namely, in $\mathrm{N}$ patients $\mathrm{Aix}_{\mathrm{ao}}$ was $9.3 \pm 7.4 \%$ whereas it was $7.6 \pm 7.0 \%$ in OW patients $(p$ $<0.00001)$, and $\mathrm{Aix}_{\mathrm{ao}}$ was higher in $\mathrm{N}$ subjects $(9.7 \pm 8.1 \%)$ compared to $\mathrm{O}$ patients $(6.6 \pm 7.2 \%)$, which difference proved to be statistically significant $(p<0.00001)$ as well. Regarding $\mathrm{SBP}_{\mathrm{ao}}$ no significant difference was found between $\mathrm{N}$ and OW (110.7 \pm $12.4 \mathrm{mmHg}$ vs. $110.3 \pm 11.9 \mathrm{mmHg}$ ) subjects. In the same way, the difference was not significant between the $\mathrm{SBP}_{\mathrm{ao}}$ values of propensity score matched $\mathrm{N}$ and $\mathrm{O}$ subjects $(115.6 \pm 14.0 \mathrm{mmHg}$ vs. $114.3 \pm 12.8 \mathrm{mmHg}$ ).

\section{DISCUSSION}

The most important finding of our study is that no difference was found regarding $\mathrm{PWV}_{\mathrm{ao}}$ between $\mathrm{N}$ and $\mathrm{OW}, \mathrm{N}$ and $\mathrm{O}$ patients after accounting for the effect of covariates. Furthermore, we observed a marked, significant decrease of $\mathrm{Aix}_{\mathrm{a} o}$ in OW, and a markedly more significant decrease in $\mathrm{O}$ patients. Finallysimilarly to $\mathrm{PWV}_{\mathrm{ao}}$-no differences were found regarding $\mathrm{SBP}_{\mathrm{ao}}$ neither in $\mathrm{OW}$, nor in $\mathrm{O}$ patients in a properly matched analysis.

\section{$\mathbf{P W V}_{\text {ao }}$}

With the use of propensity score analysis, no significant difference was found within the different weight categories regarding $\mathrm{PWV}_{\mathrm{ao}}$. This is in apparent disagreement with previous reports indicated increased $\mathrm{PWV}_{\text {ao }}$ in $\mathrm{O}$ children and adolescents $(26,27)$. Analyzing the reference values of $\mathrm{PWV}_{\mathrm{ao}}$ measured in children and adolescents (18), it is apparent that the median percentile curve of $\mathrm{PWV}_{\mathrm{ao}}$ shows a flat period between the age of three and the beginning of puberty with a steeper increase thereafter in both sexes. Therefore, it is necessary to match the age of the investigated patients and control groups precisely. Only three publications were found in which the mean age of the examined groups were identical (26-28). However, in these studies, $\mathrm{SBP}_{\text {brach }}$ differed in the different studied patients' groups, i.e., the $\mathrm{SBP}_{\text {brach }}$ was higher in the $\mathrm{OW} / \mathrm{O}$ groups compared to the controls. It is well known that the $\mathrm{PWV}_{\mathrm{ao}}$ is significantly dependent on the $\mathrm{SBP}_{\text {brach }}(20)$, namely the higher the $\mathrm{SBP}_{\text {brach, }}$, the higher the $\mathrm{PWV}_{\mathrm{ao}}$. Consequently, in these studies, the elevated $\mathrm{PWV}_{\mathrm{ao}}$ may originate from increased $\mathrm{SBP}_{\text {brach }}$. Eliminating the possible modifying factors, age, sex, and $\mathrm{SBP}_{\text {brach }}$ matched control groups were formed in our study. As a result of this, no significant differences were found regarding $\mathrm{PWV}_{\mathrm{ao}}$. Charakida et al. published data on PWV in children (over 6,000 subjects) with elevated BMI, however, they measured regional (radial) PWV, not aortic. Unfortunately, predicting the value of regional (brachial, radial, tibial) PWV is not established in cardiovascular morbidity and mortality, so it is considered irrelevant from our aspect (13). Lurbe et al. (29) published AFPs data in overweight and obese children, nevertheless, the difference in age between the investigated groups was more than 1 year, which might lead to misinterpretations in the aspect of AFPs (obese $12 \pm 2.2$ y vs. overweight $13 \pm 2.1$ y vs. non-obese $13.4 \pm 2.6 \mathrm{y})$.

\section{Aixao}

$\mathrm{Aix}_{\mathrm{ao}}$ a measure of central wave reflection, is strongly associated with peripheral arterial resistance (30), which is determined mainly by the tone of small arteries and arterioles. From the cited three studies (26-28) only Mocnik et al. measured Aix, and they found that the Aix was lower in OW/O patients than in controls (28). Unfortunately, it was not published whether Aix $_{\mathrm{ao}}$ or Aix $x_{\text {brach }}$ was measured. Aix $\mathrm{ao}_{\mathrm{a}}$ and Aix $\mathrm{x}_{\text {brach }}$ are correlated very strongly, they are basically identical, only the scaling is different between aortic and brachial Aix (24). Thus, Mocnik's findings support our observed results where we have found the Aix $_{\mathrm{a} o}$ to be significantly lower in $\mathrm{OW} / \mathrm{O}$ patients groups compared to $\mathrm{N}$ subjects.

Nevertheless, the enhanced wave reflection in small children is a well-known phenomenon (31), moreover, our working group proved that the increased $\mathrm{Aix}_{\mathrm{a}}$ detected in early childhood can only be caused by the shorter body height (aortic length) (25). Therefore, it is possible, that the observed differences regarding 
TABLE 1 | Characteristics for boys.

\begin{tabular}{|c|c|c|c|c|c|c|c|c|c|c|c|c|}
\hline $\begin{array}{l}\text { Age } \\
\text { (years) }\end{array}$ & $\mathrm{n} / \mathrm{n}_{\text {sum }}$ & $\begin{array}{l}\text { Weight } \\
\text { (kg) }\end{array}$ & $\begin{array}{l}\text { Height } \\
\text { (cm) }\end{array}$ & $\begin{array}{l}\text { BMI } \\
(\%)\end{array}$ & $\begin{array}{l}\text { SBP }_{\text {brach }} \\
(\mathrm{mmHg})\end{array}$ & $\begin{array}{l}\mathrm{DBP}_{\text {brach }} \\
(\mathrm{mmHg})\end{array}$ & $\begin{array}{c}\text { MAP } \\
(\mathrm{mmHg})\end{array}$ & $\begin{array}{c}\mathrm{PP} \\
(\mathrm{mmHg})\end{array}$ & $\begin{array}{c}\text { HR } \\
(1 / \mathrm{min})\end{array}$ & $\begin{array}{c}\mathrm{PWV}_{\mathrm{ao}} \\
(\mathrm{m} / \mathrm{s})\end{array}$ & $\begin{array}{c}A i x_{a o} \\
(\%)\end{array}$ & $\begin{array}{l}\mathrm{SBP}_{\mathrm{ao}} \\
(\mathrm{mmHg})\end{array}$ \\
\hline \multicolumn{13}{|c|}{ (A) NORMAL WEIGHT $n=2880$} \\
\hline 3 & $53 / 62$ & $15.8 \pm 2.3$ & $104.1 \pm 6.4$ & $14.6 \pm 1.7$ & $103.7 \pm 6.7$ & $61.7 \pm 7.8$ & $76.5 \pm 7.8$ & $45.5 \pm 10.3$ & $97.8 \pm 16.1$ & $5.4 \pm 0.5$ & $19.2 \pm 8.5$ & $97.4 \pm 8.0$ \\
\hline 4 & $74 / 88$ & $17.7 \pm 2.6$ & $109.2 \pm 7.3$ & $14.8 \pm 1.6$ & $104.7 \pm 8.4$ & $61.0 \pm 7.2$ & $76.4 \pm 8.1$ & $45.7 \pm 8.4$ & $92.5 \pm 12.8$ & $5.5 \pm 0.6$ & $16.1 \pm 6.4$ & $96.3 \pm 9.0$ \\
\hline 5 & $132 / 153$ & $20.0 \pm 2.7$ & $116.0 \pm 6.3$ & $14.8 \pm 1.5$ & $107.1 \pm 7.9$ & $62.8 \pm 6.1$ & $78.5 \pm 7.6$ & $46.7 \pm 9.7$ & $91.5 \pm 12.6$ & $5.4 \pm 0.6$ & $15.2 \pm 5.8$ & $98.6 \pm 9.2$ \\
\hline 6 & $193 / 222$ & $22.2 \pm 2.9$ & $122.6 \pm 5.8$ & $14.7 \pm 1.4$ & $107.1 \pm 9.6$ & $62.7 \pm 6.8$ & $77.8 \pm 7.6$ & $45.7 \pm 9.0$ & $87.0 \pm 12.4$ & $5.4 \pm 0.6$ & $13.9 \pm 5.9$ & $97.9 \pm 10.2$ \\
\hline 7 & $147 / 187$ & $24.6 \pm 3.3$ & $127.4 \pm 6.2$ & $15.1 \pm 1.4$ & $108.6 \pm 8.8$ & $63.0 \pm 6.7$ & $78.4 \pm 7.0$ & $46.6 \pm 8.5$ & $82.7 \pm 13.0$ & $5.3 \pm 0.6$ & $13.3 \pm 6.2$ & $98.7 \pm 8.9$ \\
\hline 8 & 139/180 & $27.8 \pm 4.2$ & $133.5 \pm 7.0$ & $15.6 \pm 1.5$ & $108.6 \pm 8.7$ & $63.0 \pm 6.0$ & $78.2 \pm 6.2$ & $45.5 \pm 6.7$ & $79.6 \pm 13.1$ & $5.4 \pm 0.6$ & $13.0 \pm 6.5$ & $98.2 \pm 8.7$ \\
\hline 9 & 159/199 & $31.2 \pm 5.3$ & $138.4 \pm 7.4$ & $16.2 \pm 1.7$ & $110.8 \pm 8.6$ & $65.4 \pm 6.7$ & $80.3 \pm 6.7$ & $45.9 \pm 6.2$ & $79.8 \pm 13.9$ & $5.4 \pm 0.6$ & $11.3 \pm 6.0$ & $100.0 \pm 8.7$ \\
\hline 10 & 122/181 & $35.2 \pm 5.6$ & $144.1 \pm 6.7$ & $16.9 \pm 1.8$ & $110.6 \pm 8.3$ & $64.5 \pm 5.4$ & $80.1 \pm 5.9$ & $46.5 \pm 7.9$ & $76.5 \pm 11.8$ & $5.4 \pm 0.6$ & $10.4 \pm 5.7$ & $99.6 \pm 8.0$ \\
\hline 11 & 134/195 & $37.5 \pm 6.6$ & $148.1 \pm 8.3$ & $17.0 \pm 1.9$ & $111.1 \pm 8.2$ & $64.8 \pm 6.0$ & $80.3 \pm 6.4$ & $46.6 \pm 5.9$ & $73.9 \pm 11.9$ & $5.4 \pm 0.6$ & $11.3 \pm 6.4$ & $99.9 \pm 7.9$ \\
\hline 12 & 170/232 & $43.6 \pm 8.6$ & $156.0 \pm 9.4$ & $17.8 \pm 2.1$ & $115.6 \pm 10.3$ & $65.7 \pm 6.8$ & $82.5 \pm 7.4$ & $49.9 \pm 7.8$ & $77.3 \pm 14.5$ & $5.6 \pm 0.7$ & $8.1 \pm 6.3$ & $102.2 \pm 8.9$ \\
\hline 13 & $245 / 331$ & $50.0 \pm 8.7$ & $163.5 \pm 9.5$ & $18.6 \pm 1.9$ & $120.2 \pm 10.7$ & $66.4 \pm 6.7$ & $84.5 \pm 7.3$ & $53.9 \pm 9.1$ & $76.9 \pm 13.8$ & $5.7 \pm 0.7$ & $5.8 \pm 5.3$ & $104.7 \pm 9.0$ \\
\hline 14 & $301 / 410$ & $55.8 \pm 9.1$ & $170.4 \pm 9.3$ & $19.1 \pm 1.9$ & $123.5 \pm 11.3$ & $67.6 \pm 6.3$ & $86.3 \pm 7.2$ & $55.8 \pm 8.8$ & $75.4 \pm 13.9$ & $6.0 \pm 0.7$ & $5.0 \pm 5.7$ & $107.2 \pm 10.1$ \\
\hline 15 & $304 / 374$ & $59.6 \pm 8.5$ & $174.3 \pm 7.7$ & $19.6 \pm 2.0$ & $125.0 \pm 11.8$ & $68.9 \pm 7.1$ & $87.7 \pm 8.0$ & $56.1 \pm 9.2$ & $72.2 \pm 13.1$ & $6.0 \pm 0.6$ & $4.9 \pm 5.4$ & $108.6 \pm 10.4$ \\
\hline 16 & $288 / 351$ & $63.0 \pm 8.9$ & $176.6 \pm 7.3$ & $20.2 \pm 2.2$ & $129.0 \pm 13.0$ & $70.5 \pm 7.3$ & $90.0 \pm 8.5$ & $58.5 \pm 9.5$ & $73.3 \pm 15.0$ & $6.2 \pm 0.6$ & $5.2 \pm 6.0$ & $111.9 \pm 11.0$ \\
\hline 17 & 295/356 & $65.6 \pm 8.8$ & $178.3 \pm 7.0$ & $20.6 \pm 2.2$ & $130.1 \pm 12.9$ & $70.3 \pm 7.2$ & $90.2 \pm 8.1$ & $59.6 \pm 10.4$ & $71.4 \pm 13.2$ & $6.3 \pm 0.7$ & $5.2 \pm 5.8$ & $112.5 \pm 10.9$ \\
\hline \multirow[t]{2}{*}{18} & $124 / 147$ & $68.2 \pm 8.2$ & $179.5 \pm 6.7$ & $21.1 \pm 2.0$ & $131.7 \pm 11.5$ & $68.2 \pm 7.6$ & $89.4 \pm 7.5$ & $63.1 \pm 10.5$ & $71.4 \pm 13.8$ & $6.4 \pm 0.5$ & $5.3 \pm 5.1$ & $113.0 \pm 9.9$ \\
\hline & $2880 / 3668$ & & & & & & & & & & & \\
\hline \multicolumn{13}{|c|}{$\mathrm{HT} n=516$} \\
\hline 3 & $8 / 62$ & $18.4 \pm 2.1$ & $101.3 \pm 6.5$ & $17.9 \pm 0.3$ & $105.0 \pm 7.4$ & $61.0 \pm 5.3$ & $75.9 \pm 6.1$ & $44.9 \pm 5.8$ & $98.3 \pm 10.7$ & $5.4 \pm 0.8$ & $14.2 \pm 7.0$ & $95.9 \pm 7.5$ \\
\hline 4 & $12 / 88$ & $23.1 \pm 4.9$ & $111.7 \pm 11.5$ & $18.3 \pm 0.5$ & $111.0 \pm 9.0$ & $64.5 \pm 7.1$ & $81.6 \pm 10.1$ & $51.3 \pm 11.9$ & $89.8 \pm 10.9$ & $5.1 \pm 0.6$ & $14.5 \pm 6.5$ & $104.1 \pm 14.5$ \\
\hline 5 & $13 / 153$ & $25.3 \pm 3.4$ & $117.4 \pm 6.7$ & $18.3 \pm 0.7$ & $113.6 \pm 8.5$ & $65.8 \pm 6.3$ & $82.2 \pm 6.7$ & $49.3 \pm 8.9$ & $87.9 \pm 12.5$ & $5.3 \pm 0.6$ & $15.6 \pm 7.2$ & $102.7 \pm 7.3$ \\
\hline 6 & $12 / 222$ & $29.7 \pm 4.0$ & $127.0 \pm 8.1$ & $18.3 \pm 0.5$ & $112.5 \pm 13.6$ & $62.9 \pm 6.7$ & $79.4 \pm 8.3$ & $49.6 \pm 9.8$ & $85.3 \pm 10.5$ & $5.2 \pm 0.5$ & $11.2 \pm 5.1$ & $99.5 \pm 10.6$ \\
\hline 7 & $26 / 187$ & $33.0 \pm 3.3$ & $131.7 \pm 6.4$ & $19.0 \pm 0.7$ & $116.5 \pm 9.3$ & $68.2 \pm 5.1$ & $84.6 \pm 6.2$ & $49.1 \pm 7.2$ & $87.6 \pm 15.5$ & $5.3 \pm 0.7$ & $12.1 \pm 6.9$ & $105.0 \pm 8.7$ \\
\hline 8 & $24 / 180$ & $36.9 \pm 4.6$ & $136.3 \pm 7.6$ & $19.8 \pm 0.9$ & $112.9 \pm 7.5$ & $65.9 \pm 4.6$ & $81.5 \pm 5.2$ & $47.0 \pm 5.0$ & $80.2 \pm 10.6$ & $5.2 \pm 0.6$ & $9.5 \pm 5.2$ & $100.6 \pm 6.8$ \\
\hline 9 & 20/199 & $41.5 \pm 4.3$ & $141.2 \pm 6.6$ & $20.8 \pm 1.0$ & $117.7 \pm 7.8$ & $65.9 \pm 4.7$ & $83.2 \pm 5.1$ & $51.8 \pm 6.6$ & $76.6 \pm 9.4$ & $5.4 \pm 0.5$ & $11.5 \pm 4.7$ & $105.0 \pm 7.0$ \\
\hline 10 & $29 / 181$ & $49.4 \pm 5.1$ & $148.7 \pm 6.5$ & $22.3 \pm 1.1$ & $119.0 \pm 9.0$ & $68.5 \pm 7.4$ & $85.2 \pm 6.4$ & $51.3 \pm 9.4$ & $82.7 \pm 14.0$ & $5.6 \pm 0.8$ & $8.3 \pm 6.7$ & $105.9 \pm 10.1$ \\
\hline 11 & 39/195 & $53.1 \pm 7.3$ & $152.7 \pm 8.9$ & $22.7 \pm 1.4$ & $121.5 \pm 10.2$ & $68.5 \pm 6.9$ & $86.1 \pm 7.4$ & $53.0 \pm 7.2$ & $75.6 \pm 13.3$ & $5.6 \pm 0.8$ & $8.7 \pm 6.4$ & $107.4 \pm 8.5$ \\
\hline 12 & 39/232 & $60.4 \pm 8.0$ & $159.8 \pm 8.8$ & $23.6 \pm 1.5$ & $122.6 \pm 7.9$ & $67.8 \pm 6.5$ & $86.4 \pm 6.2$ & $55.6 \pm 9.3$ & $78.1 \pm 13.2$ & $5.7 \pm 0.7$ & $5.3 \pm 4.3$ & $107.1 \pm 7.8$ \\
\hline 13 & $48 / 331$ & $67.6 \pm 7.9$ & $166.6 \pm 8.1$ & $24.3 \pm 1.5$ & $126.5 \pm 10.8$ & $66.9 \pm 8.8$ & $87.0 \pm 9.3$ & $60.3 \pm 9.4$ & $75.1 \pm 10.3$ & $6.0 \pm 0.7$ & $6.6 \pm 7.5$ & $110.8 \pm 13.1$ \\
\hline 14 & $81 / 410$ & $74.2 \pm 8.9$ & $171.3 \pm 8.9$ & $25.2 \pm 1.3$ & $131.3 \pm 12.9$ & $68.9 \pm 7.7$ & $89.7 \pm 8.2$ & $62.4 \pm 11.1$ & $76.0 \pm 14.2$ & $6.3 \pm 0.7$ & $4.4 \pm 6.0$ & $112.6 \pm 10.5$ \\
\hline 15 & $52 / 374$ & $77.5 \pm 7.7$ & $174.9 \pm 7.1$ & $25.3 \pm 1.4$ & $134.9 \pm 13.5$ & $70.7 \pm 6.4$ & $92.1 \pm 7.6$ & $64.2 \pm 11.7$ & $73.0 \pm 11.3$ & $6.1 \pm 0.7$ & $5.5 \pm 7.7$ & $116.6 \pm 12.1$ \\
\hline 16 & $46 / 351$ & $82.5 \pm 7.6$ & $178.0 \pm 6.9$ & $26.0 \pm 1.1$ & $135.0 \pm 13.2$ & $71.8 \pm 7.9$ & $92.9 \pm 9.1$ & $63.5 \pm 9.5$ & $70.9 \pm 11.6$ & $6.2 \pm 0.6$ & $3.9 \pm 6.4$ & $116.4 \pm 12.7$ \\
\hline 17 & $46 / 356$ & $83.8 \pm 8.6$ & $178.3 \pm 8.3$ & $26.3 \pm 1.1$ & $137.1 \pm 13.2$ & $72.2 \pm 7.6$ & $94.0 \pm 9.5$ & $65.5 \pm 10.4$ & $72.5 \pm 12.5$ & $6.5 \pm 0.6$ & $4.7 \pm 6.2$ & $118.3 \pm 12.3$ \\
\hline \multirow[t]{2}{*}{18} & $21 / 147$ & $85.1 \pm 10.6$ & $178.3 \pm 8.5$ & $26.7 \pm 1.4$ & $143.2 \pm 13.3$ & $74.6 \pm 8.4$ & $97.6 \pm 9.2$ & $68.6 \pm 9.6$ & $77.0 \pm 16.5$ & $6.8 \pm 0.6$ & $5.0 \pm 4.1$ & $122.9 \pm 11.9$ \\
\hline & $516 / 3668$ & & & & & & & & & & & \\
\hline
\end{tabular}


TABLE 1 | Continued

\begin{tabular}{|c|c|c|c|c|c|c|c|c|c|c|c|c|}
\hline $\begin{array}{l}\text { Age } \\
\text { (years) }\end{array}$ & $n / n_{\text {sum }}$ & $\begin{array}{c}\text { Weight } \\
\text { (kg) }\end{array}$ & $\begin{array}{l}\text { Height } \\
\text { (cm) }\end{array}$ & $\begin{array}{l}\text { BMI } \\
(\%)\end{array}$ & $\begin{array}{l}\text { SBP }_{\text {brach }} \\
(\mathrm{mmHg})\end{array}$ & $\begin{array}{l}\text { DBP }_{\text {brach }} \\
(\mathrm{mmHg})\end{array}$ & $\begin{array}{c}\text { MAP } \\
(\mathrm{mmHg})\end{array}$ & $(\mathrm{mmHg})$ & $\begin{array}{c}H R \\
(1 / \mathrm{min})\end{array}$ & $\begin{array}{l}P W V_{a o} \\
(\mathrm{~m} / \mathrm{s})\end{array}$ & $\begin{array}{l}\text { Aixao } \\
(\%)\end{array}$ & $\begin{array}{l}\mathrm{SBP}_{\mathrm{ao}} \\
(\mathrm{mmHg})\end{array}$ \\
\hline \multicolumn{13}{|c|}{ (C) OBESE $n=272$} \\
\hline 3 & $1 / 62$ & $21.0 \pm N A$ & $104.0 \pm N A$ & $19.4 \pm N A$ & $111.0 \pm N A$ & $65.0 \pm N A$ & $80.0 \pm N A$ & $46.0 \pm N A$ & $110.0 \pm N A$ & $5.1 \pm \mathrm{NA}$ & $9.7 \pm \mathrm{NA}$ & $99.0 \pm N A$ \\
\hline 4 & $2 / 88$ & $27.5 \pm 2.1$ & $117.5 \pm 3.5$ & $19.9 \pm 0.3$ & $119 \pm 21.2$ & $56.5 \pm 5.0$ & $77.5 \pm 3.5$ & $62.5 \pm 26.2$ & $84.5 \pm 16.3$ & $4.8 \pm 0.1$ & $9.6 \pm 1.8$ & $102.4 \pm 13.4$ \\
\hline 5 & $8 / 153$ & $34.5 \pm 4.3$ & $123.6 \pm 4.1$ & $22.6 \pm 2.4$ & $128.5 \pm 16.0$ & $64.1 \pm 8.1$ & $86.6 \pm 9.8$ & $68.1 \pm 18.2$ & $95.0 \pm 16.9$ & $6.0 \pm 0.7$ & $10.4 \pm 4.5$ & $114.9 \pm 15.3$ \\
\hline 6 & $17 / 222$ & $34.8 \pm 4.5$ & $124.9 \pm 4.5$ & $22.2 \pm 1.6$ & $116.9 \pm 10.4$ & $62.4 \pm 7.1$ & $81.0 \pm 7.2$ & $55.7 \pm 13.1$ & $85.5 \pm 13.0$ & $5.4 \pm 0.5$ & $9.6 \pm 5.0$ & $103.4 \pm 9.4$ \\
\hline 7 & $14 / 187$ & $43.8 \pm 5.5$ & $135.8 \pm 6.3$ & $23.7 \pm 1.9$ & $123.6 \pm 12.2$ & $67.0 \pm 6.5$ & $85.9 \pm 7.2$ & $56.6 \pm 11.3$ & $83.6 \pm 13.6$ & $5.3 \pm 0.6$ & $10.0 \pm 6.7$ & $109.4 \pm 10.5$ \\
\hline 8 & $17 / 180$ & $49.5 \pm 8.4$ & $139.6 \pm 8.4$ & $25.3 \pm 2.5$ & $120.6 \pm 7.8$ & $70.0 \pm 4.3$ & $87.0 \pm 4.5$ & $50.7 \pm 7.7$ & $81.2 \pm 11.3$ & $5.5 \pm 0.7$ & $9.6 \pm 5.3$ & $107.3 \pm 5.5$ \\
\hline 9 & 20/199 & $55.2 \pm 6.6$ & $145.6 \pm 6.4$ & $26.0 \pm 1.8$ & $124.5 \pm 10.4$ & $69.4 \pm 4.3$ & $87.9 \pm 5.1$ & $55.1 \pm 10.0$ & $86.4 \pm 11.7$ & $5.8 \pm 0.6$ & $6.7 \pm 6.9$ & $109.2 \pm 8.3$ \\
\hline 10 & $30 / 181$ & $63.2 \pm 9.6$ & $149.7 \pm 7.2$ & $28.2 \pm 3.6$ & $124.3 \pm 10.5$ & $68.3 \pm 7.5$ & $87.1 \pm 8.2$ & $56.3 \pm 7.5$ & $81.7 \pm 14.1$ & $5.7 \pm 0.7$ & $5.0 \pm 4.4$ & $108.8 \pm 9.3$ \\
\hline 11 & 22/195 & $70.8 \pm 8.6$ & $157.1 \pm 7.5$ & $28.6 \pm 2.5$ & $130.0 \pm 10.8$ & $72.3 \pm 7.3$ & $91.6 \pm 7.1$ & $57.7 \pm 10.6$ & $77.3 \pm 12.3$ & $5.9 \pm 0.6$ & $5.2 \pm 4.5$ & $112.9 \pm 8.5$ \\
\hline 12 & 23/232 & $80.3 \pm 8.9$ & $162.7 \pm 5.6$ & $30.3 \pm 3.0$ & $130.0 \pm 9.5$ & $67.7 \pm 6.6$ & $88.5 \pm 6.5$ & $62.2 \pm 8.9$ & $81.2 \pm 14.6$ & $6.0 \pm 0.5$ & $3.3 \pm 3.3$ & $110.7 \pm 7.7$ \\
\hline 13 & $38 / 331$ & $85.3 \pm 11.0$ & $167.0 \pm 7.2$ & $30.5 \pm 2.6$ & $134.1 \pm 14.5$ & $68.3 \pm 8.8$ & $90.3 \pm 9.4$ & $66.0 \pm 12.7$ & $82.8 \pm 10.6$ & $6.3 \pm 0.8$ & $3.4 \pm 8.6$ & $114.5 \pm 12.2$ \\
\hline 14 & $28 / 410$ & $91.9 \pm 16.0$ & $170.6 \pm 9.5$ & $31.4 \pm 3.0$ & $132.0 \pm 14.5$ & $69.9 \pm 9.0$ & $90.6 \pm 10.1$ & $62.1 \pm 10.0$ & $75.7 \pm 8.9$ & $6.4 \pm 0.6$ & $4.1 \pm 7.8$ & $113.8 \pm 12.2$ \\
\hline 15 & $18 / 374$ & $97.7 \pm 10.6$ & $173.7 \pm 5.9$ & $32.3 \pm 3.1$ & $146.3 \pm 15.1$ & $76.9 \pm 9.4$ & $99.9 \pm 10.5$ & $69.4 \pm 9.8$ & $72.4 \pm 13.7$ & $6.6 \pm 0.8$ & $4.0 \pm 4.4$ & $125.3 \pm 13.1$ \\
\hline 16 & $17 / 351$ & $101.9 \pm 10.9$ & $176.3 \pm 6.4$ & $32.8 \pm 3.2$ & $145.8 \pm 15.8$ & $78.1 \pm 10.5$ & $101.3 \pm 13.1$ & $69.5 \pm 10.7$ & $76.3 \pm 13.9$ & $6.5 \pm 0.9$ & $7.2 \pm 5.8$ & $128.2 \pm 17.1$ \\
\hline 17 & $15 / 356$ & $104.8 \pm 10.5$ & $179.3 \pm 6.2$ & $32.6 \pm 2.5$ & $146.8 \pm 16.1$ & $77.8 \pm 7.8$ & $100.9 \pm 9.3$ & $69.0 \pm 13.6$ & $75.3 \pm 16.6$ & $6.7 \pm 0.8$ & $4.4 \pm 5.9$ & $125.7 \pm 12.3$ \\
\hline \multirow[t]{2}{*}{18} & $2 / 147$ & $89.0 \pm 7.1$ & $171.0 \pm 5.7$ & $30.4 \pm 0.4$ & $153.0 \pm 19.8$ & $84.0 \pm 4.2$ & $107.0 \pm 9.9$ & $69.0 \pm 15.6$ & $72.0 \pm 4.2$ & $5.8 \pm 0.0$ & $8.1 \pm 1.9$ & $134.2 \pm 16.6$ \\
\hline & $272 / 3668$ & & & & & & & & & & & \\
\hline
\end{tabular}

Data are reported as mean $\pm S D$. Aix ao, aortic augmentation index; BMI, body mass index; $D B P_{\text {brach, }}$, brachial diastolic blood pressure; HR, heart rate; MAP, mean arterial blood pressure; PP, pulse pressure; PWVao, aortic pulse wave velocity; $S B P_{a o}$, aortic systolic blood pressure; $S B P_{\text {brach, }}$ brachial systolic blood pressure. 
TABLE 2 | Characteristics for girls.

\begin{tabular}{|c|c|c|c|c|c|c|c|c|c|c|c|c|}
\hline $\begin{array}{l}\text { Age } \\
\text { (years) }\end{array}$ & $n / n_{\text {sum }}$ & $\begin{array}{c}\text { Weight } \\
\text { (kg) }\end{array}$ & $\begin{array}{l}\text { Height } \\
\text { (cm) }\end{array}$ & $(\%)$ & $\begin{array}{l}\text { SBP }_{\text {brach }} \\
(\mathrm{mmHg})\end{array}$ & $\begin{array}{l}\mathrm{DBP}_{\text {brach }} \\
(\mathrm{mmHg})\end{array}$ & (mmHg) & $(\mathrm{mmHg})$ & $\begin{array}{c}\text { HR } \\
(1 / \mathrm{min})\end{array}$ & $\begin{array}{l}\mathrm{PWV}_{\mathrm{ao}} \\
(\mathrm{m} / \mathrm{s})\end{array}$ & $\begin{array}{l}\text { Aix }_{a \circ} \\
(\%)\end{array}$ & $\begin{array}{l}\mathrm{SBP}_{\mathrm{ao}} \\
(\mathrm{mmHg})\end{array}$ \\
\hline \multicolumn{13}{|c|}{ (A) NORMAL WEIGHT $n=2580$} \\
\hline 3 & $41 / 49$ & $15.5 \pm 2.5$ & $102.0 \pm 6.4$ & $14.8 \pm 1.2$ & $103.4 \pm 8.8$ & $59.8 \pm 9.7$ & $74.8 \pm 8.0$ & $47.2 \pm 13.2$ & $101.8 \pm 13.9$ & $5.6 \pm 0.7$ & $21.8 \pm 8.2$ & $97.7 \pm 10.0$ \\
\hline 4 & $66 / 87$ & $17.1 \pm 2.5$ & $108.7 \pm 6.5$ & $14.5 \pm 1.7$ & $106.1 \pm 7.7$ & $61.2 \pm 6.2$ & $76.9 \pm 6.6$ & $46.5 \pm 7.9$ & $93.5 \pm 11.7$ & $5.4 \pm 0.6$ & $18.7 \pm 6.2$ & $99.5 \pm 9.5$ \\
\hline 5 & $82 / 99$ & $18.5 \pm 3.0$ & $114.3 \pm 7.3$ & $14.2 \pm 1.6$ & $107.7 \pm 11.4$ & $62.6 \pm 8.0$ & $78.2 \pm 9.2$ & $47.0 \pm 9.6$ & $94.4 \pm 11.1$ & $5.5 \pm 0.7$ & $16.3 \pm 6.8$ & $99.6 \pm 12.5$ \\
\hline 6 & $123 / 148$ & $21.7 \pm 3.4$ & $122.4 \pm 6.9$ & $14.5 \pm 1.5$ & $109.3 \pm 11.1$ & $63.5 \pm 7.3$ & $79.2 \pm 8.7$ & $46.8 \pm 8.3$ & $90.1 \pm 14.2$ & $5.4 \pm 0.7$ & $15.3 \pm 6.6$ & $100.1 \pm 11.5$ \\
\hline 7 & $116 / 142$ & $23.9 \pm 4.9$ & $127.6 \pm 7.9$ & $14.6 \pm 1.9$ & $110.1 \pm 10.8$ & $64.5 \pm 7.4$ & $80.2 \pm 8.9$ & $46.8 \pm 9.0$ & $87.0 \pm 13.0$ & $5.5 \pm 0.6$ & $14.5 \pm 7.3$ & $101.1 \pm 12.1$ \\
\hline 8 & $94 / 122$ & $27.4 \pm 4.8$ & $133.2 \pm 7.4$ & $15.3 \pm 1.7$ & $111.1 \pm 10.1$ & $64.9 \pm 6.2$ & $80.2 \pm 7.0$ & $46.1 \pm 7.0$ & $85.0 \pm 13.5$ & $5.4 \pm 0.6$ & $13.1 \pm 5.9$ & $100.5 \pm 9.2$ \\
\hline 9 & $123 / 157$ & $29.7 \pm 5.1$ & $137.6 \pm 7.4$ & $15.6 \pm 1.7$ & $111.9 \pm 10.1$ & $65.2 \pm 6.4$ & $80.6 \pm 7.1$ & $47.1 \pm 8.2$ & $85.2 \pm 13.0$ & $5.5 \pm 0.7$ & $11.1 \pm 5.1$ & $100.5 \pm 9.2$ \\
\hline 10 & $111 / 151$ & $34.2 \pm 5.8$ & $143.9 \pm 7.0$ & $16.4 \pm 1.9$ & $113.2 \pm 9.9$ & $65.4 \pm 5.9$ & $81.4 \pm 6.7$ & $47.7 \pm 7.0$ & $83.2 \pm 12.7$ & $5.6 \pm 0.7$ & $10.7 \pm 6.3$ & $101.5 \pm 8.6$ \\
\hline 11 & $104 / 137$ & $38.2 \pm 7.3$ & $149.1 \pm 9.4$ & $17.0 \pm 2.0$ & $113.7 \pm 9.0$ & $66.4 \pm 5.9$ & $82.1 \pm 6.4$ & $47.4 \pm 6.7$ & $81.6 \pm 13.7$ & $5.5 \pm 0.7$ & $9.7 \pm 5.7$ & $101.5 \pm 8.1$ \\
\hline 12 & $162 / 218$ & $43.3 \pm 7.6$ & $155.7 \pm 7.8$ & $17.7 \pm 2.0$ & $117.7 \pm 12.2$ & $66.9 \pm 6.9$ & $83.9 \pm 8.1$ & $50.9 \pm 8.7$ & $82.4 \pm 15.5$ & $5.8 \pm 0.6$ & $8.9 \pm 6.5$ & $104.1 \pm 10.2$ \\
\hline 13 & $257 / 317$ & $49.1 \pm 6.7$ & $161.4 \pm 6.9$ & $18.8 \pm 2.0$ & $119.4 \pm 11.7$ & $67.0 \pm 6.5$ & $84.5 \pm 7.3$ & $52.5 \pm 9.5$ & $80.5 \pm 14.8$ & $5.9 \pm 0.7$ & $7.5 \pm 5.6$ & $104.9 \pm 10.1$ \\
\hline 14 & $301 / 356$ & $52.0 \pm 7.4$ & $164.0 \pm 6.6$ & $19.3 \pm 2.2$ & $119.9 \pm 11.2$ & $68.2 \pm 6.5$ & $85.5 \pm 7.4$ & $51.7 \pm 8.3$ & $78.1 \pm 14.4$ & $5.9 \pm 0.6$ & $7.4 \pm 5.3$ & $105.6 \pm 9.7$ \\
\hline 15 & $316 / 374$ & $54.1 \pm 7.5$ & $164.8 \pm 6.7$ & $19.9 \pm 2.2$ & $119.0 \pm 10.6$ & $68.8 \pm 6.3$ & $85.5 \pm 7.3$ & $50.2 \pm 7.5$ & $77.4 \pm 13.8$ & $5.9 \pm 0.6$ & $7.8 \pm 5.4$ & $105.3 \pm 9.1$ \\
\hline 16 & $281 / 335$ & $54.7 \pm 7.2$ & $164.9 \pm 6.5$ & $20.1 \pm 2.3$ & $120.1 \pm 10.5$ & $70.2 \pm 6.1$ & $86.9 \pm 7.1$ & $50.0 \pm 7.7$ & $77.1 \pm 13.7$ & $6.1 \pm 0.6$ & $8.3 \pm 5.6$ & $106.7 \pm 9.5$ \\
\hline 17 & $271 / 311$ & $55.4 \pm 7.4$ & $166.1 \pm 6.4$ & $20.1 \pm 2.2$ & $121.2 \pm 11.7$ & $69.4 \pm 6.7$ & $86.7 \pm 7.6$ & $51.8 \pm 8.8$ & $76.9 \pm 12.1$ & $6.2 \pm 0.6$ & $8.6 \pm 5.8$ & $107.2 \pm 10.2$ \\
\hline \multirow[t]{2}{*}{18} & $132 / 145$ & $56.7 \pm 6.5$ & $166.0 \pm 6.7$ & $20.6 \pm 2.0$ & $125.5 \pm 13.8$ & $70.9 \pm 7.6$ & $89.1 \pm 9.1$ & $54.6 \pm 9.5$ & $79.6 \pm 13.1$ & $6.4 \pm 0.5$ & $8.1 \pm 5.4$ & $110.6 \pm 11.9$ \\
\hline & $2580 / 3148$ & & & & & & & & & & & \\
\hline \multicolumn{13}{|c|}{ (B) OVERWEIGHT $n=395$} \\
\hline 3 & $6 / 49$ & $18.7 \pm 3.2$ & $100.5 \pm 7.9$ & $18.4 \pm 0.6$ & $112.3 \pm 6.7$ & $62.3 \pm 3.7$ & $79.5 \pm 4.3$ & $51.7 \pm 11.1$ & $104.7 \pm 8.8$ & $5.2 \pm 0.6$ & $18.9 \pm 7.3$ & $105.0 \pm 10.2$ \\
\hline 4 & $11 / 87$ & $20.9 \pm 3.6$ & $107.8 \pm 8.8$ & $17.9 \pm 0.4$ & $109.6 \pm 8.2$ & $62.3 \pm 6.5$ & $78.2 \pm 6.2$ & $47.7 \pm 7.6$ & $96.6 \pm 17.4$ & $5.2 \pm 0.6$ & $14.8 \pm 7.2$ & $99.5 \pm 5.8$ \\
\hline 5 & $11 / 99$ & $27.4 \pm 3.6$ & $122.4 \pm 8.5$ & $18.2 \pm 0.7$ & $118.2 \pm 9.8$ & $69.0 \pm 11.9$ & $86.6 \pm 12.7$ & $52.6 \pm 8.0$ & $94.7 \pm 18.5$ & $5.3 \pm 0.7$ & $10.9 \pm 7.6$ & $107.9 \pm 15.4$ \\
\hline 6 & $18 / 148$ & $29.8 \pm 4.2$ & $126.4 \pm 8.2$ & $18.6 \pm 0.8$ & $112.9 \pm 12.1$ & $65.1 \pm 8.5$ & $81.2 \pm 9.3$ & $48.4 \pm 7.2$ & $85.2 \pm 13.2$ & $5.3 \pm 0.5$ & $14.1 \pm 8.3$ & $102.3 \pm 11.0$ \\
\hline 7 & $17 / 142$ & $32.2 \pm 2.6$ & $131.1 \pm 4.6$ & $18.7 \pm 0.5$ & $114.9 \pm 8.7$ & $64.0 \pm 5.6$ & $81.1 \pm 5.2$ & $50.9 \pm 9.2$ & $85.6 \pm 8.9$ & $5.2 \pm 0.7$ & $10.8 \pm 7.8$ & $102.5 \pm 9.4$ \\
\hline 8 & $15 / 122$ & $35.9 \pm 5.0$ & $134.3 \pm 6.7$ & $19.8 \pm 1.1$ & $114.9 \pm 10.1$ & $66.6 \pm 4.8$ & $82.6 \pm 5.9$ & $48.3 \pm 8.0$ & $79.2 \pm 11.9$ & $5.2 \pm 0.6$ & $12.4 \pm 6.6$ & $103.7 \pm 7.6$ \\
\hline 9 & $22 / 157$ & $40.5 \pm 5.2$ & $139.3 \pm 8.1$ & $20.8 \pm 0.8$ & $117.8 \pm 10.9$ & $66.0 \pm 5.9$ & $83.2 \pm 6.9$ & $51.8 \pm 8.1$ & $83.5 \pm 13.9$ & $5.2 \pm 0.8$ & $9.7 \pm 7.0$ & $104.4 \pm 7.7$ \\
\hline 10 & $21 / 151$ & $47.8 \pm 4.5$ & $147.7 \pm 6.1$ & $21.9 \pm 1.0$ & $121.1 \pm 13.6$ & $67.5 \pm 8.0$ & $85.7 \pm 10.0$ & $54.6 \pm 8.5$ & $89.0 \pm 16.1$ & $5.5 \pm 0.9$ & $5.5 \pm 3.7$ & $106.1 \pm 12.0$ \\
\hline 11 & $22 / 137$ & $56.1 \pm 8.1$ & $155.0 \pm 8.9$ & $23.2 \pm 1.4$ & $125.2 \pm 11.4$ & $66.5 \pm 9.6$ & $86.4 \pm 10.0$ & $59.7 \pm 9.8$ & $86.4 \pm 15.4$ & $6.0 \pm 0.8$ & $5.2 \pm 5.9$ & $108.5 \pm 11.8$ \\
\hline 12 & $40 / 218$ & $63.5 \pm 7.0$ & $161.0 \pm 6.6$ & $24.5 \pm 1.7$ & $127.3 \pm 11.4$ & $70.5 \pm 7.0$ & $89.6 \pm 8.1$ & $57.2 \pm 8.5$ & $84.4 \pm 13.9$ & $5.9 \pm 0.7$ & $6.0 \pm 4.0$ & $111.4 \pm 10.5$ \\
\hline 13 & $51 / 317$ & $64.2 \pm 7.6$ & $160.4 \pm 9.8$ & $24.9 \pm 1.4$ & $127.5 \pm 13.4$ & $70.3 \pm 7.5$ & $89.4 \pm 8.8$ & $57.2 \pm 9.6$ & $82.3 \pm 13.5$ & $5.9 \pm 0.7$ & $7.1 \pm 6.2$ & $112.0 \pm 11.6$ \\
\hline 14 & $43 / 356$ & $69.0 \pm 7.6$ & $163.9 \pm 7.2$ & $25.6 \pm 1.4$ & $129.0 \pm 13.4$ & $71.3 \pm 7.6$ & $90.5 \pm 8.7$ & $57.7 \pm 10.3$ & $79.1 \pm 13.4$ & $6.0 \pm 0.7$ & $7.4 \pm 10.3$ & $113.1 \pm 11.8$ \\
\hline 15 & $41 / 374$ & $70.4 \pm 6.7$ & $165.0 \pm 6.7$ & $25.8 \pm 1.3$ & $125.5 \pm 11.2$ & $71.6 \pm 6.3$ & $89.5 \pm 7.2$ & $54.0 \pm 9.2$ & $75.3 \pm 10.6$ & $6.0 \pm 0.7$ & $7.6 \pm 4.7$ & $110.7 \pm 10.0$ \\
\hline 16 & $34 / 335$ & $73.0 \pm 6.7$ & $165.8 \pm 6.6$ & $26.5 \pm 1.2$ & $131.1 \pm 12.6$ & $74.1 \pm 7.6$ & $93.0 \pm 8.3$ & $57.0 \pm 9.8$ & $79.5 \pm 13.1$ & $6.1 \pm 0.7$ & $6.7 \pm 6.4$ & $115.2 \pm 11.4$ \\
\hline 17 & $33 / 311$ & $75.5 \pm 7.8$ & $166.6 \pm 6.9$ & $27.1 \pm 1.6$ & $130.9 \pm 14.1$ & $72.2 \pm 7.6$ & $91.8 \pm 9.1$ & $58.8 \pm 10.0$ & $74.1 \pm 9.6$ & $6.1 \pm 0.5$ & $8.2 \pm 5.3$ & $115.1 \pm 12.3$ \\
\hline \multirow[t]{2}{*}{18} & $10 / 145$ & $76.6 \pm 7.0$ & $166.3 \pm 7.6$ & $27.7 \pm 1.6$ & $125.9 \pm 10.8$ & $71.5 \pm 3.7$ & $89.6 \pm 5.7$ & $54.4 \pm 8.6$ & $86.5 \pm 8.5$ & $6.4 \pm 0.6$ & $6.6 \pm 2.8$ & $110.4 \pm 8.7$ \\
\hline & $395 / 3148$ & & & & & & & & & & & \\
\hline
\end{tabular}


TABLE 2 | Continued

\begin{tabular}{|c|c|c|c|c|c|c|c|c|c|c|c|c|}
\hline $\begin{array}{l}\text { Age } \\
\text { (years) }\end{array}$ & $n / n_{\text {sum }}$ & $\begin{array}{c}\text { Weight } \\
\text { (kg) }\end{array}$ & $\begin{array}{l}\text { Height } \\
\text { (cm) }\end{array}$ & $\begin{array}{l}\text { BMI } \\
(\%)\end{array}$ & $\begin{array}{l}\text { SBP }_{\text {brach }} \\
(\mathrm{mmHg})\end{array}$ & $\begin{array}{l}\text { DBP }_{\text {brach }} \\
(\mathrm{mmHg})\end{array}$ & $\begin{array}{c}\text { MAP } \\
(\mathrm{mmHg})\end{array}$ & $(\mathrm{mmHg})$ & $\begin{array}{c}H R \\
(1 / \mathrm{min})\end{array}$ & $\begin{array}{l}\mathrm{PWV}_{\mathrm{ao}} \\
(\mathrm{m} / \mathrm{s})\end{array}$ & $\begin{array}{l}\text { Aixao } \\
(\%)\end{array}$ & $\begin{array}{l}\mathrm{SBP}_{\mathrm{ao}} \\
(\mathrm{mmHg})\end{array}$ \\
\hline \multicolumn{13}{|c|}{ (C) OBESE $n=173$} \\
\hline 3 & $2 / 49$ & $21.5 \pm 0.7$ & $100.5 \pm 0.7$ & $21.3 \pm 1.0$ & $127 \pm 4.2$ & $73.0 \pm 2.8$ & $91.0 \pm 2.8$ & $54.0 \pm 1.4$ & $97.5 \pm 12.0$ & $5.9 \pm 0.5$ & $13.1 \pm 8.9$ & $114.6 \pm 0.3$ \\
\hline 4 & $10 / 87$ & $30.4 \pm 4.0$ & $113.6 \pm 7.1$ & $23.5 \pm 2.4$ & $120.7 \pm 7.0$ & $63.5 \pm 5.4$ & $82.5 \pm 5.2$ & $57.2 \pm 6.3$ & $99.0 \pm 10.3$ & $5.9 \pm 0.7$ & $10.2 \pm 10.4$ & $106.3 \pm 7.0$ \\
\hline 5 & $6 / 99$ & $29.3 \pm 5.3$ & $117.0 \pm 8.3$ & $21.3 \pm 1.9$ & $113.2 \pm 10.1$ & $63.2 \pm 4.8$ & $79.7 \pm 5.7$ & $50.0 \pm 8.6$ & $90.0 \pm 11.1$ & $5.4 \pm 0.6$ & $16.9 \pm 11.5$ & $104.5 \pm 14.6$ \\
\hline 6 & $7 / 148$ & $30.6 \pm 3.2$ & $118.7 \pm 4.2$ & $21.6 \pm 1.1$ & $112.1 \pm 9.4$ & $63.6 \pm 5.6$ & $79.6 \pm 6.0$ & $48.6 \pm 8.5$ & $89.0 \pm 7.4$ & $5.4 \pm 0.4$ & $12.0 \pm 6.2$ & $100.4 \pm 7.5$ \\
\hline 7 & $9 / 142$ & $50.2 \pm 11.2$ & $128.9 \pm 5.5$ & $30.7 \pm 8.7$ & $119.0 \pm 10.2$ & $66.3 \pm 7.2$ & $83.8 \pm 6.5$ & $52.7 \pm 10.9$ & $88.0 \pm 5.9$ & $5.6 \pm 0.6$ & $6.5 \pm 5.6$ & $104.2 \pm 8.8$ \\
\hline 8 & 13/122 & $49.9 \pm 4.3$ & $141.7 \pm 3.5$ & $24.9 \pm 1.8$ & $122.8 \pm 8.9$ & $69.2 \pm 5.6$ & $87.2 \pm 6.1$ & $53.6 \pm 6.7$ & $87.5 \pm 6.7$ & $5.5 \pm 0.5$ & $7.7 \pm 4.8$ & $107.7 \pm 7.1$ \\
\hline 9 & $12 / 157$ & $54.1 \pm 8.4$ & $144.0 \pm 8.7$ & $26.0 \pm 2.0$ & $126.5 \pm 12.4$ & $70.1 \pm 7.6$ & $89.0 \pm 8.4$ & $56.4 \pm 10.0$ & $84.3 \pm 10.8$ & $5.7 \pm 0.8$ & $9.7 \pm 5.4$ & $112.0 \pm 10.6$ \\
\hline 10 & 19/151 & $69.5 \pm 10.7$ & $154.7 \pm 8.5$ & $29.0 \pm 3.3$ & $140.2 \pm 14.5$ & $73.4 \pm 6.1$ & $95.7 \pm 8.1$ & $66.8 \pm 11.8$ & $94.2 \pm 14.5$ & $6.1 \pm 0.8$ & $4.2 \pm 9.5$ & $121.2 \pm 12.6$ \\
\hline 11 & $11 / 137$ & $69.2 \pm 8.7$ & $156.8 \pm 8.4$ & $28.1 \pm 1.8$ & $125.9 \pm 10.4$ & $72.3 \pm 9.1$ & $90.3 \pm 9.3$ & $53.6 \pm 6.0$ & $79.3 \pm 15.6$ & $5.8 \pm 0.8$ & $9.0 \pm 7.5$ & $111.8 \pm 12.7$ \\
\hline 12 & $16 / 218$ & $80.6 \pm 12.2$ & $160.6 \pm 7.1$ & $31.4 \pm 5.5$ & $132.1 \pm 14.2$ & $70.6 \pm 7.7$ & $91.1 \pm 8.2$ & $61.4 \pm 13.2$ & $81.1 \pm 15.4$ & $5.9 \pm 0.9$ & $9.2 \pm 13.0$ & $116.2 \pm 12.1$ \\
\hline 13 & $9 / 317$ & $78.6 \pm 11.0$ & $162.2 \pm 10.4$ & $29.7 \pm 1.3$ & $135.7 \pm 6.2$ & $71.3 \pm 8.2$ & $92.8 \pm 6.8$ & $64.3 \pm 7.9$ & $86.1 \pm 6.7$ & $6.5 \pm 0.5$ & $8.4 \pm 10.8$ & $118.9 \pm 9.1$ \\
\hline 14 & $12 / 356$ & $84.6 \pm 11.4$ & $164.1 \pm 6.7$ & $31.3 \pm 3.0$ & $140.9 \pm 19.3$ & $76.3 \pm 11.3$ & $97.8 \pm 13.3$ & $64.6 \pm 11.4$ & $87.4 \pm 9.2$ & $6.6 \pm 0.7$ & $4.4 \pm 5.4$ & $121.8 \pm 16.7$ \\
\hline 15 & $17 / 374$ & $90.6 \pm 11.6$ & $166.5 \pm 7.1$ & $32.6 \pm 2.6$ & $137.4 \pm 11.9$ & $74.9 \pm 5.7$ & $95.7 \pm 7.3$ & $62.4 \pm 8.5$ & $79.1 \pm 11.5$ & $6.4 \pm 0.7$ & $6.5 \pm 6.6$ & $119.7 \pm 8.9$ \\
\hline 16 & 20/335 & $92.5 \pm 11.8$ & $165.9 \pm 6.0$ & $33.6 \pm 3.7$ & $138.9 \pm 10.5$ & $78.3 \pm 3.8$ & $98.6 \pm 5.5$ & $60.7 \pm 8.2$ & $81.6 \pm 12.1$ & $6.4 \pm 0.5$ & $8.6 \pm 6.4$ & $122.5 \pm 8.9$ \\
\hline 17 & $7 / 311$ & $94.7 \pm 10.5$ & $161.7 \pm 9.9$ & $36.2 \pm 2.1$ & $147.6 \pm 14.7$ & $73.3 \pm 8.7$ & $98.0 \pm 7.7$ & $74.3 \pm 17.0$ & $80.1 \pm 14.7$ & $6.2 \pm 0.8$ & $5.3 \pm 3.6$ & $125.7 \pm 10.4$ \\
\hline \multirow[t]{2}{*}{18} & $3 / 145$ & $102.0 \pm 0.0$ & $169.0 \pm 0.0$ & $35.7 \pm 0.0$ & $133.7 \pm 15.0$ & $62.0 \pm 9.5$ & $86 \pm 11.1$ & $71.7 \pm 5.8$ & $57.7 \pm 1.2$ & $5.6 \pm 0.4$ & $1.3 \pm 4.0$ & $110.8 \pm 13.5$ \\
\hline & $173 / 3148$ & & & & & & & & & & & \\
\hline
\end{tabular}

Data are reported as mean $\pm S D$. Aix ao, aortic augmentation index; BMI, body mass index; $D B P_{\text {brach, }}$ brachial diastolic blood pressure; HR, heart rate; MAP, mean arterial blood pressure; PP, pulse pressure; PWVao, aortic pulse wave velocity; $S B P_{a o}$, aortic systolic blood pressure; $S B P_{\text {brach, }}$ brachial systolic blood pressure. 
TABLE 3 | Mean heart rate and $\mathrm{SBP}_{\text {brach }}$ values in N/OW/O patients.

\begin{tabular}{|c|c|c|c|c|c|c|}
\hline & $\mathbf{N}$ & OW & 0 & N-OW* & OW-O* & $\mathrm{N}-\mathrm{O}^{*}$ \\
\hline Number of patients ( $n$ ) & 5460 & 911 & 445 & & & \\
\hline $\begin{array}{l}\text { Heart rate } \\
(1 / \mathrm{min})\end{array}$ & $79.5 \pm 14.9$ & $79.6 \pm 14.1$ & $82.5 \pm 13.5$ & $\begin{array}{c}\text { NS } \\
(p=0.98)\end{array}$ & $p=0.02$ & $p<0.0001$ \\
\hline $\begin{array}{l}\text { SBP }_{\text {brach }} \\
(\mathrm{mmHg})\end{array}$ & $117.7 \pm 13.1$ & $125.6 \pm 14.0$ & $131.2 \pm 15.2$ & $p<0.0001$ & $p<0.0001$ & $p<0.0001$ \\
\hline
\end{tabular}

Data are reported as mean $\pm S D$. HR, heart rate; N, normal weight; O, obese; OW, overweight; SBPbrach, brachial systolic blood pressure; *, Kruskal-Wallis test.

TABLE 4 | Characteristics of OW and O patients vs. controls and AFP values after propensity score matching.

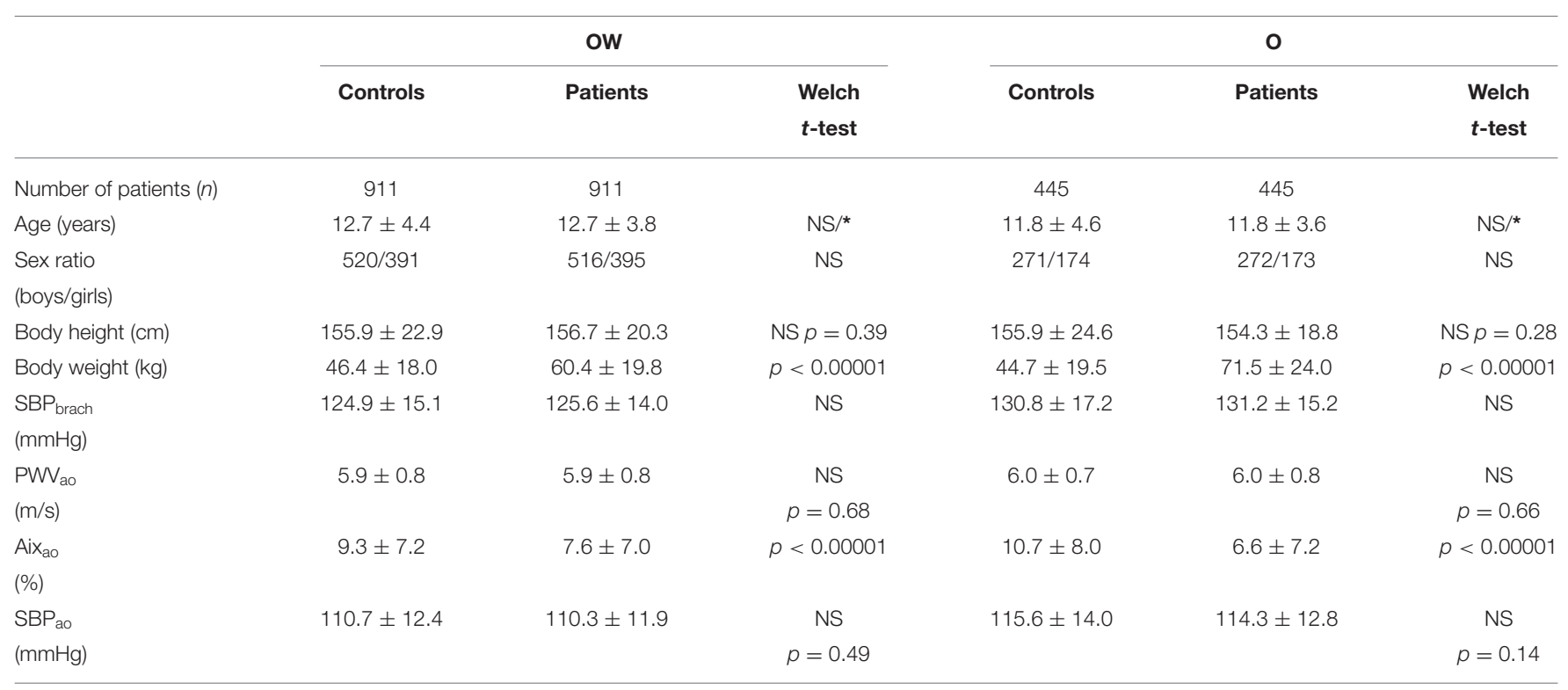

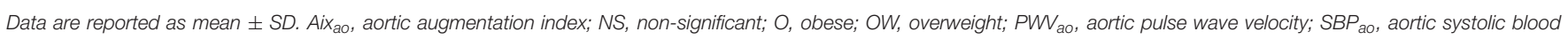
pressure; $\mathrm{SBP}_{\text {brach, }}$ brachial systolic blood pressure; * , mean age was significantly different between $\mathrm{OW}$ and $\mathrm{O}$ group, $p<0.001$.

Aix $_{\mathrm{ao}}$ between $\mathrm{N}$, OW and $\mathrm{O}$ groups might stem from the differences in body height. As the mean body height was not significantly different between patients and controls-neither in case of $\mathrm{OW}$, nor in $\mathrm{O}$-we might declare that the observed differences regarding $\mathrm{Aix}_{\mathrm{ao}}$ are independent of the body height.

Obesity in children and adolescents is characterized by chronic sympathetic overdrive (19). Sympathetic overactivity occurs in obese animals, as evidenced by increased blood pressure, cardiac output, heart rate as well as the activation of the renin-angiotensin-aldosterone system (32). In our study

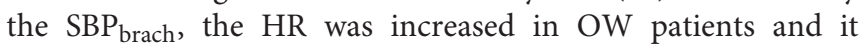
was significantly higher in $\mathrm{O}$ subjects than in controls. This finding could be explained by the previously mentioned chronic sympathetic overdrive; consequently, the increased metabolism will cause a remarkable increase in heart rate, stroke volume and cardiac output. Wilkinson et al. have found an inverse linear relationship between $\mathrm{HR}$ and $\mathrm{Aix}_{\mathrm{ao}}$ (33). Together these findings could explain the observed lower $\mathrm{Aix}_{\mathrm{ao}}$ in $\mathrm{OW} / \mathrm{O}$ patients. Presumably, due to the increased cardiac output and the concomitantly increasing $\mathrm{SBP}_{\text {brach }}$ and $\mathrm{SBP}_{\mathrm{ao}}$, the lateral tension toward the aortic wall is also increased, the aorta dilates; thus, the aortic wall becomes stiffer and, as its consequence, the $P W V_{a}$ could be higher. To compensate for the effects of increased cardiac output and $\mathrm{SBP}_{\mathrm{ao}}$, the vascular regulation system might lower the vascular resistance by dilating the small arterioles; therefore, decreased $\mathrm{Aix}_{\mathrm{ao}}$ could be detected. This finding might be supported by the observations, which revealed increased nitric oxide (NO) level in relation to fat accumulation (34).

\section{SBP}

In the overviewed and cited literature $\mathrm{SBP}_{\mathrm{a}}$ has not been measured or published. In our study, after the elimination of the effects of sex, age, and $\mathrm{SBP}_{\text {brach, no significant differences }}$ were found regarding $\mathrm{SBP}_{\mathrm{a}}$ in $\mathrm{OW} / \mathrm{O}$ patients and controls. This observation might originate from the accurately matched $\mathrm{SBP}_{\text {brach }}$ (age and sex also) between patient and control groups, consequently, in this age period, the $\mathrm{SBP}_{\mathrm{ao}}$ is mainly determined by $\mathrm{SBP}_{\text {brach }}$, without any detectable morphological or functional deterioration of the aortic wall. 


\section{Study Limitations}

Although the use of BMI is well-known and widely accepted technique to identify overweight and obesity, it might be misleading in some cases, because it does not distinguish fat tissue from muscle tissue, therefore individuals with a considerable muscular system may have increased BMI resulting in "artificial" obesity. In our study, age- and sex-based BMI was used to categories patients, still, other techniques such as skinfold measurements, waist-hip ratio, or bioelectrical impedance analysis would be necessary for more proper weight categorization.

To confirm our theory according to which the sympathetic overdrive in OW/O patients resulting in increased cardiac output and elevated $\mathrm{SBP}_{\text {brach }}$, stroke volume and cardiac output should have been measured by echocardiography in these subjects. In our study, there is a lack of these data, since it was not the aim of this investigation. Detailed history taking on overweight and obesity (onset, duration) and medical history about the closest family members could add relevant information to this study. Finally, there is a lack of lifestyle data (smoking, caffeine intake, eating habits, etc) and detailed laboratory tests (fatty-acid profile, metabolic panel). Future studies of this working group will collect the above-mentioned data in order to elucidate the underlying causes of the observed phenomena and hypothesis.

\section{CONCLUSIONS}

Our study comprises anthropometric and AFPs data of a huge cohort of N/OW/O subjects. Interestingly, by comparing OW/O population data with the properly matched identical subset of $N$ subjects, we found that $\mathrm{OW} / \mathrm{O}$ in children and adolescents was not associated with increased aortic stiffness. Based on our results, we may conclude that the pathophysiological consequences in the circulatory system due to childhood OW/O are partially compensated hemodynamically in these patients, presumably by the opening of small arterioles, by decreasing peripheral arteriolar resistance. Therefore, considering our findings we might speculate that OW/O does not have any direct adverse effect on the arterial function in the by us investigated population. However, it must be emphasized, that in general accumulation of adipose tissue raises the blood pressure-as we also presented in this study-resulting in increased cardiovascular risk in the future. Hence, successful lifestyle intervention programs (focusing on weight reduction, cessation of a sedentary lifestyle, sleeping, stress elimination, and relaxation) may lead to the optimization of the $\mathrm{CV}$

\section{REFERENCES}

1. Ng M, Fleming T, Robinson M, Thomson B, Graetz N, Margono C, et al. Global, regional, and national prevalence of overweight and obesity in children and adults during 1980-2013: A systematic analysis for the Global Burden of Disease Study 2013. Lancet. (2014) 384:766-81. doi: 10.1016/S0140-6736(14)60460-8 risks in the affected subjects. Furthermore, it would be important to follow these OW/O patients by monitoring AFPs, to establish the possible period, when the "haemodynamic compensation/adaptation" of the arterial system might switch into deterioration, resulting in early arterial disease.

The moment, when the compensatory functions in $\mathrm{OW} / \mathrm{O}$ subjects become insufficient is hardly detectable. For this reason, we aim to design further studies to detect the beginning of this unfavorable vascular function changes.

\section{DATA AVAILABILITY STATEMENT}

The datasets generated for this study are available on request to the corresponding author.

\section{ETHICS STATEMENT}

The studies involving human participants were reviewed and approved by Institutional Ethics Committee of the University of Pécs. Written informed consent to participate in this study was provided by the participants' legal guardian/next of kin.

\section{AUTHOR CONTRIBUTIONS}

All authors listed have made a substantial, direct and intellectual contribution to the work, and approved it for publication.

\section{FUNDING}

$\mathrm{CB}, \mathrm{AJ}, \mathrm{ZM}$, and TK were provided by the National Research, Development and Innovation Office (Hungary, EFOP-3.6.1-162016-00008). The state funders played no role in the study design, data collection and analysis, decision to publish, or in the preparation of the manuscript.

\section{ACKNOWLEDGMENTS}

The authors would like to thank István Raskó for comments that greatly improved the manuscript and Gábor Érsek for assistance with data management.

\section{SUPPLEMENTARY MATERIAL}

The Supplementary Material for this article can be found online at: https://www.frontiersin.org/articles/10.3389/fped. 2020.00389/full\#supplementary-material 
to 67 risk factors and risk factor clusters in 21 regions, 1990-2010: a systematic analysis for the Global Burden of Disease Study 2010. Lancet. (2012) 380:2224-60. doi: 10.1016/S0140-6736(12)61766-8

4. Reilly JJ, Kelly J. Long-term impact of overweight and obesity in childhood and adolescence on morbidity and premature mortality in adulthood: systematic review. Int J Obes. (2011) 35:891-8. doi: 10.1038/ijo.2010.222

5. Mancia G, Fagard R, Narkiewicz K, Redán J, Zanchetti A, Böhm $\mathrm{M}$, et al. 2013 Practice guidelines for the management of arterial hypertension of the European Society of Hypertension (ESH) and the European Society of Cardiology (ESC). J Hypertens. (2013) 31:1925-38. doi: 10.1097/HJH.0b013e328364ca4c

6. London GM, Blacher J, Pannier B, Guérin AP, Marchais SJ, Safar ME. Arterial wave reflections and survival in end-stage renal failure. Hypertens. (2001) 38:434-8. doi: 10.1161/01.HYP.38.3.434

7. Roman MJ, Devereux RB, Kizer JR, Lee ET, Galloway JM, Ali T, et al. Central pressure more strongly relates to vascular disease and outcome than does brachial pressure: the Strong Heart Study. Hypertens. (2007) 50:197-203. doi: 10.1161/HYPERTENSIONAHA.107.089078

8. Vlachopoulos C, Aznaouridis K, Stefanadis C. Prediction of cardiovascular events and all-cause mortality with arterial stiffness: a systematic review and meta-analysis. J Am Coll Cardiol. (2010) 55:1318-27. doi: 10.1016/j.jacc.2009.10.061

9. Hudson LD, Rapala A, Khan T, Williams B, Viner RM. Evidence for contemporary arterial stiffening in obese children and adolescents using pulse wave velocity: A systematic review and meta-analysis. Atherosclerosis. (2015) 241:376-86. doi: 10.1016/j.atherosclerosis.2015.05.014

10. Jin Y, Chen Y, Tang Q, Xue M, Li W, Jiang J. Evaluation of carotid artery stiffness in obese children using ultrasound radiofrequency data technology. J Ultrasound Med. (2013) 32:105-13. doi: 10.7863/jum.2013.32.1.105

11. Cabrera-Rego JO, Iacobellis G, Castillo-Herrera JA, Valiente-Mustelier J, Gandarilla-Sarmientos JC, Marín-Juliá SM, et al. Epicardial fat thickness correlates with carotid intima-media thickness, arterial stiffness, and cardiac geometry in children and adolescents. Pediatr Cardiol. (2014) 35:450-6. doi: 10.1007/s00246-013-0799-9

12. Martínez-Costa C, Núñez F, Montal A, Brines J. Relationship between childhood obesity cut-offs and metabolic and vascular comorbidities: comparative analysis of three growth standards. J Hum Nutr Diet. (2014) 27 Suppl 2:75-83. doi: 10.1111/jhn. 12140

13. Charakida M, Jones A, Falaschetti E, Khan $T$, Finer N, Sattar N, et al. Childhood obesity and vascular phenotypes: A population study. J Am Coll Cardiol. (2012) 60:2643-50. doi: 10.1016/j.jacc.2012.08.1017

14. Dangardt F, Chen Y, Berggren K, Osika W, Friberg P. Increased rate of arterial stiffening with obesity in adolescents: a five-year follow-up study. PLoS ONE. (2013) 8:e57454. doi: 10.1371/journal.pone.0057454

15. Montero D, Walther G, Perez-Martin A, Mercier CS, Roche E, Vinet A. Leg arterial stiffness after weight loss in severely obese adolescents. Int J Cardiol. (2013) 168:1676-7. doi: 10.1016/j.ijcard.2013.03.081

16. Pannier B, Guérin AP, Marchais SJ, Safar ME, London GM. Stiffness of capacitive and conduit arteries: prognostic significance for end-stage renal disease patients. Hypertens. (2005) 45:592-6. doi: 10.1161/01.HYP.0000159190.71253.c3

17. Reusz GS, Cseprekal O, Temmar M, Kis É, Cherif AB, Thaleb A, et al. Reference values of pulse wave velocity in healthy children and teenagers. Hypertension. (2010) 56:217-24. doi: 10.1161/HYPERTENSIONAHA.110.152686

18. Hidvégi EV, Illyés $M$, Benczúr $B$, Böcskei RM, Rátgéber L, Lenkey $Z$, et al. Reference values of aortic pulse wave velocity in a large healthy population aged between 3 and 18 years. J Hypertens. (2012) 30:2314-21. doi: 10.1097/HJH.0b013e328359562c

19. Qi Z, Ding S. Obesity-associated sympathetic overactivity in children and adolescents: the role of catecholamine resistance in lipid metabolism. J Pediatr Endocrinol Metab. (2016) 29:113-25. doi: 10.1515/jpem-2015-0182

20. Urbina EM, Williams R V., Alpert BS, Collins RT, Daniels SR, Hayman L, et al. Noninvasive assessment of subclinical atherosclerosis in children and adolescents: Recommendations for standard assessment for clinical research: A scientific statement from the american heart association. Hypertension. (2009) 54:919-50. doi: 10.1161/HYPERTENSIONAHA.109.192639
21. WHO. Physical Status: the Use and Interpretation of Anthropometry: Report of WHO Expert Committee. Geneva (1995).

22. Cole TJ, Lobstein T. Extended international (IOTF) body mass index cutoffs for thinness, overweight and obesity. Pediatr Obes. (2012) 7:284-94. doi: 10.1111/j.2047-6310.2012.00064.x

23. Baulmann J, Schillings U, Rickert S, Uen S, Düsing R, Illyes M, et al. A new oscillometric method for assessment of arterial stiffness: comparison with tonometric and piezo-electronic methods. J Hypertens. (2008) 26:523-8. doi: 10.1097/HJH.0b013e3282f314f7

24. Horváth IG, Németh A, Lenkey Z, Alessandri N, Tufano F, Kis P, et al. Invasive validation of a new oscillometric device (Arteriograph) for measuring augmentation index, central blood pressure and aortic pulse wave velocity. J Hypertens. (2010) 28:2068-75. doi: 10.1097/HJH.0b013e32833 c8ala

25. Hidvégi E V., Illyés M, Molnár FT, Cziráki A. Influence of body height on aortic systolic pressure augmentation and wave reflection in childhood. J Hum Hypertens. (2015) 29:495-501. doi: 10.1038/jhh.2014.118

26. Harris KC, Al Saloos HA, De Souza AM, Sanatani S, Hinchliffe M, Potts JE, et al. Biophysical properties of the aorta and left ventricle and exercise capacity in obese children. Am J Cardiol. (2012) 110:897-901. doi: 10.1016/j.amjcard.2012.05.019

27. Hacihamdioglu B, Öçal G, Berberoglu M, Siklar Z, Fitöz S, Tutar E, et al. Preperitoneal fat tissue may be associated with arterial stiffness in obese adolescents. Ultrasound Med Biol. (2014) 40:871-6. doi: 10.1016/j.ultrasmedbio.2013.11.014

28. Močnik M, Nikolić S, Varda NM. Arterial Compliance Measurement In Overweight And Hypertensive Children. Indian J Pediatr. (2016) 83:510-6. doi: 10.1007/s12098-015-1965-2

29. Lurbe E, Torro I, Garcia-vicent C, Alvarez J, Fernández-fornoso JA, Redon J. Blood pressure and obesity exert independent influences on pulse wave velocity in youth. Hypertens. (2012) 112:550-5. doi: 10.1161/HYPERTENSIONAHA.112.194746

30. Wilenius M, Tikkakoski AJ, Tahvanainen AM, Haring A, Koskela J, Huhtala H, et al. Central wave reflection is associated with peripheral arterial resistance in addition to arterial stiffness in subjects without antihypertensive medication. BMC Cardiovasc Disord. (2016) 16:1-11. doi: 10.1186/s12872-0160303-6

31. Murakami T, Takeda A, Takei K, Ueno M, Yakuwa S, Yamazawa H et al. Aortic pressure wave reflection in children. Hypertens Res. (2010) 33:225-8. doi: 10.1038/hr.2009.218

32. Hall JE, da Silva AA, do Carmo JM, Dubinion J, Hamza S, Munusamy $\mathrm{S}$, et al. Obesity-induced hypertension: role of sympathetic nervous system, leptin, and melanocortins. J Biol Chem. (2010) 285:17271-6. doi: $10.1074 /$ jbc.R110.113175

33. Wilkinson IB, MacCallum H, Flint L, Cockcroft JR, Newby DE, Webb DJ. The influence of heart rate on augmentation index and central arterial pressure in humans. J Physiol. (2000) 525:263-70. doi: 10.1111/j.1469-7793.2000.t01-100263.x

34. Correia-Costa L, Sousa T, Morato M, Cosme D, Afonso J, Areias JC, et al. Oxidative stress and nitric oxide are increased in obese children and correlate with cardiometabolic risk and renal function. Br J Nutr. (2016) 116:805-15. doi: $10.1017 /$ S0007114516002804

Conflict of Interest: MI shares a part in TensioMed Company (Budapest, Hungary), which produces Arteriograph and other medical devices.

The remaining authors declare that the research was conducted in the absence of any commercial or financial relationships that could be construed as a potential conflict of interest.

Copyright (c) 2020 Jakab, Hidvégi, Illyés, Cziráki, Kalmár, Maróti and Bereczki. This is an open-access article distributed under the terms of the Creative Commons Attribution License (CC BY). The use, distribution or reproduction in other forums is permitted, provided the original author(s) and the copyright owner(s) are credited and that the original publication in this journal is cited, in accordance with accepted academic practice. No use, distribution or reproduction is permitted which does not comply with these terms. 